Economical estimation of interns and residents of general surgery from Universidad Surcolombiana to the general surgery Department of University Hospital Hernando Moncaleano Perdomo in Neiva

\section{Resumen}

Objetivo. En este estudio se cuantificó el valor de las ayudantías quirúrgicas, entre mayo de 2008 y abril de 2009, de estudiantes del pregrado de medicina y de diferentes post-grados clínicos de la Universidad Surcolombiana al Hospital Universitario Hernando Moncaleano Perdomo. Se incluyó el apoyo brindado en: revista de piso, atención de urgencias, consulta externa de Cirugía General y las subespecialidades obtenidas por la intervención.

Materiales y métodos. Se revisó la estadística reportada a la Superintendencia Nacional de Salud, Subgerencias, Hospitalización, Coordinación de Cirugía y Urgencias, agendas del personal de planta y contrato, los horarios y funcionamiento de los Internos y Residentes. Se cruzó, filtró y ajustó la información en tablas de Microsoft Office Excel 2007. Para la fecha del estudio, el valor de la hora médica corresponde a $\$ 42.500$ y el de las ayudantías a la facturación y contratación vigente a tarifas ISS 2001 y SOAT 2008-2009.

Resultados. Se cuantifica un aporte mínimo total anual de $\$ 601.734 .830$ con ayudantías quirúrgicas a tarifa ISS 2001 o \$ 644.566 .046 con ayudantías quirúrgicas a tarifas SOAT 2008-2009, que se discrimina en \$159.778.750 revista de piso, $\$ 62.050 .000$ cirugía de tórax, $\$ 62.050 .000$ gastro-oncología, \$93.075.000 soporte metabólico y nutricional, \$10.625.000 clínica de heridas y \$214.156,080 ayudantías quirúrgicas ISS 2001 o \$256.987.296 ayudantías quirúrgicas SOAT 2008-2009.

Conclusión. El convenio docencia-asistencia se convierte, en lo referente a Cirugía General, en una fortaleza y una oportunidad.

Cirujano General Fellow UCI-FSFB. Coordinador Departamento de Cirugía USCO-HUN. Profesor Asistente, Departamento de Ciencias Clínicas, Facultad de Salud, Universidad Surcolombiana. Correo electrónico: lesanabriar@gmail.com.

* Presentado en el XXXVI Congreso de Avances en Cirugía ACC Bogotá, 2010. 
Palabras clave: personal médico, Economía Hospitalaria, Administración Hospitalaria, práctica médica.

\begin{abstract}
Objective: this study estimated the value of surgical assistantships, between May, 2008 and April, 2009; of undergraduate medicine students and different clinical postgraduate studies from Universidad Surcolombiana to University Hospital Hernando Moncaleano Perdomo. The support given by floor review, emergency service, general surgery outpatient and sub-specialties obtained by the surgery.
\end{abstract}

Materials and Methods: it was checked the statistical data reported to the National Health Administration, assistance management, hospitalization, surgery and emergenciy coordination, permanent and contract staff agenda, schedule and duties of interns and residents. Data were crossed, filtered and adjusted in tables of Microsof Office Excel 2007. By the date of the study, a medical hour is worth $\$ 42.500$ and an assistantship hour at existing billing and hiring ISS 2001 and SOAT 2008-2009.

Results: a minimum total annual contribution of $\$ 601.734 .830$ is quantified coming from surgical assistantships at ISS 2001 rate or $\$ 644.566 .046$ coming from surgical assistantships at SOAT 2008-2009 rate which is divided into $\$ 159.778 .750$ floor review, $\$ 62.050 .000$ thoracic surgery, \$62.050.000 gastrointestinal-oncology, \$93.075.000 metabolic and nutritional support, $\$ 10.625 .000$ injure clinics and $\$ 214.156 .080$ surgical assistantships ISS 2001 or \$256.987.296 surgical assistantships SOAT 2008-2009.

Conclusion: the teaching-assistantship agreement represents, regarding General surgery, a strength and an opportunity.

Key words: medical staff, hospital economy, hospital administration, Physician's Internship.

\section{INTRODUCCIÓN}

La administración del Hospital Universitario Hernando Moncaleano Perdomo de la ciudad de Neiva (HUN) tradicionalmente ha cuestionado el aporte económico de la Universidad Surcolombiana (USCO) y ve en el convenio docencia asistencia una debilidad y no una oportunidad.

No se discute el aporte docente, científico e investigativo que aportan el pregrado y los postgrados de la USCO al HUN que se refleja en aumento de la calidad en la atención y generan prestigio institucional.

Los costos agregados a los servicios de atención a los pacientes derivados de las actividades de docencia, investigación y extensión ha llevado a que los hospitales universitarios sean considerados, en condiciones estándar de operación, más costosos que los otros tipos de hospitales (costo cama/día, costo estancia, costo diagnóstico y tratamiento, costo tiempos quirúrgicos, etc.), este fenómeno es reconocido en estudios internacionales, y en Colombia por el Centro de Investigaciones para Desarrollo-CID de la Facultad de Ciencias Económicas de la Universidad Nacional ${ }^{(1-5)}$.

El Sistema Obligatorio de Garantía de la Calidad SOGC, conjunto de normas, instituciones, requisitos, mecanismos y procesos orientados a generar, mantener y mejorar la calidad de los servicios de salud en el país, desarrolla parcialmente la garantía de los derechos fundamentales de la Constitución Política de Colombia del 1991, limita la enseñanza tradicional obligándola a garantizar la seguridad del paciente, definida como la reducción de la ocurrencia de eventos adversos ${ }^{(6)}$

Todo este marco debe reflejarse en los convenios Docencia-Asistencia, reglamentados mediante el decreto 190 de 1996 e inmersos en el Sistema General de Seguridad Social en Salud SGSSS y específicamente la ley 100 de $1993^{(7)}$.

Estudios previos han mostrado la importancia de este convenio entre las dos instituciones $(8,9)$, no obstante la metodología y enfoque allí utilizado no es aplicable actualmente.

Se pretende entonces, en el periodo comprendido entre el 1 de mayo de 2008 y el 30 de abril de 2009 , cuantificar formalmente el valor de las ayudantías quirúrgicas, de las revistas de piso, de la atención de urgencias, consulta externa y demás actividades en las que intervienen, estudiantes de pregrado, post-grado y docentes de la Universidad Surcolombiana en la E.S.E. HUN, Nivel atención III, con 350 camas de las cuales 36 corresponden a cirugía general en piso y 55 en observación y urgencias de acuerdo al reporte actualizado a 1 de junio de $2009^{(8)}$

Una vez valorado se podrá determinar objetivamente si el aporte de la USCO al HUN puede considerarse como una debilidad, amenaza, fortaleza u oportunidad.

\section{MATERIALES Y MÉTODOS}

Se revisó la información estadística proporcionada por la oficina encargada de los reportes a 
la Superintendencia Nacional de Salud, Subgerencias de planeación y desarrollo Institucional, técnico-científica, de servicios ambulatorios, jefatura de hospitalización, comité de cancelaciones, coordinación departamental de Cirugía General, las agendas del personal de planta y a contrato, por parte del HUN y de la agendas de planta y cátedra, horarios, asignación de funciones, horarios de los Internos y Residentes por parte de la USCO.

Fue necesario hacer una revisión de los libros de registro manual de consultas de Urgencias y Externa, Interconsultas, Cuidados Intensivos, Cirugía gastrointestinal, mama, cabeza y cuello, tórax, trasplantes, cirugía programada y de Urgencias, cruzar información y ajustarla a lo realmente ejecutado, minimizando los sesgos pertinentes.

Para la valoración económica se obtuvo la información de las Subgerencias Financiera, Administrativa, Técnico-científica, Jefatura de Hospitalización, Coordinación de Cirugía General del HUN y de la Cooperativa de Trabajo Asociado que los agrupa. Para la fecha evaluada el valor de la hora correspondía a cuarenta y dos mil quinientos pesos $\$ 42.500,00$ y el valor de facturación y contratación vigente tarifa ISS 2001 y SOAT 2009.

Se revisó la estadística del servicio, egresos/año, kardex diario, urgencias/año, reporte diario de las actividades y ayudantías y cirugías de los residentes e internos y se confronto con los informes a la Superintendencia Nacional de Salud de la oficina de estadística y la jefatura de hospitalización del HUN y los informes escritos (libros) de cirugía y de interconsultas.

En todos los casos se trato, "por lo bajo" de identificar el aporte de la USCO a las labores asistenciales del HUN, procurando en todos los casos revisar y asignar las tarifas que efectivamente pueden atribuirse a la participación de los internos y residentes en los procesos pertinentes.

Para cada una de las actividades se tuvo en cuenta el funcionamiento del servicio, de la revista de piso mañana y tarde, urgencias, consulta externa, cirugía programada y de urgencias, se revisaron las subespecialidades, cuadro de turnos $\mathrm{y}$ agendas de cirujano, internos y residentes.

\section{RESULTADOS}

Revista de piso. Se revisaron y cruzaron los horarios de internos y residentes con la contratación del HUN, se revisaron la cantidad de egresos y se realizaron los cálculos.

Por tanto para un promedio de 43 pacientes/ día en piso, el mínimo aporte de la USCO, sin tener en cuenta que en la noche permanece un interno en piso, es de \$159.778.750,00 al año (Tablas 1 y 2).

Urgencias. Se revisaron los horarios de internos y residentes comparados con el cirujano del HUN contratado las 24 horas del día (Tablas 3-5).

En la atención de Urgencias no es posible afirmar que se realice un aporte distinto al de la ayudantía quirúrgica, no así en la calidad y oportunidad en la atención que se deriva del cubrimiento que realiza el personal que queda a cargo mientras el equipo quirúrgico se encuentra en quirófanos, del apoyo en la reanimación, en la atención, recepción simultanea de pacientes, de la recepción y apoyo en la toma de laboratorios e imágenes diagnósticas, reclamación de resultados y de las de secretaría en el llenado de historias, ingreso, evolución y epicrisis.

Tabla 1. Cuantificación horaria de la revista de piso en el Hospital Universitario Hernando Moncaleano Perdomo, entre mayo de 2008 y abril de 2009.

\begin{tabular}{|c|c|c|c|c|c|c|c|c|c|c|c|c|c|}
\hline Revista piso/hora & 06 & 07 & 08 & 09 & 10 & 11 & 12 & 13 & 14 & 15 & 16 & 17 & 18 \\
\hline Interno & & & & & & & & & & & & & \\
\hline Residente & & & & & & & & & & & & & \\
\hline Cirujano & & & & & & & & & & & & & \\
\hline
\end{tabular}


Tabla 2. Cuantificación económica de la revista de piso en el Hospital Universitario Hernando Moncaleano Perdomo, entre mayo de 2008 y abril de 2009.

\begin{tabular}{ll}
\hline \multicolumn{1}{c}{ Descripción } & \multicolumn{1}{c}{ Resultado } \\
\hline 2.462 egresos / año x 6.43 días estancia / 365 días/año & 43.37 pacientes/día \\
43 pacientes / día / 3 pacientes/hora AM & 14,3 horas/día \\
+ 2 horas revista de internos y residentes PM & 2,0 horas \\
Subtotal horas de revista/día & 16,3 horas \\
Hospital Universitario de Neiva & 6,0 horas \\
Universidad Surcolombiana & 10,3 horas \\
HUN: \$ 42.500,00 x 6.0 horas x 365 días/año & $\$ 93.075 .000,00$ \\
\hline USCO: \$ 42.500,00 x 10,3 horas x 365 días/año & $\mathbf{\$ 1 5 9 . 7 7 8 . 7 5 0 , 0 0}$ \\
\hline
\end{tabular}

Tabla 3. Cuantificación horaria del servicio de urgencias del Hospital Universitario Hernando Moncaleano Perdomo, de las 07 a las 18 horas entre mayo de 2008 y abril de 2009.

\begin{tabular}{|c|c|c|c|c|c|c|c|c|c|c|c|c|}
\hline Urgencias/hora & 07 & 08 & 09 & 10 & 11 & 12 & 13 & 14 & 15 & 16 & 17 & 18 \\
\hline Interno & & & & & & & & & & & & \\
\hline Residente & & & & & & & & & & & & \\
\hline Cirujano & & & & & & & & & 册 & & 册冊 & \\
\hline
\end{tabular}

Tabla 4. Cuantificación horaria del servicio de urgencias del Hospital Universitario Hernando Moncaleano Perdomo, de las 19 a las 06 horas entre mayo de 2008 y abril de 2009.

\begin{tabular}{|c|c|c|c|c|c|c|c|c|c|c|c|c|}
\hline Urgencias/hora & 19 & 20 & 21 & 22 & 23 & 24 & 01 & 02 & 03 & 04 & 05 & 06 \\
\hline Interno & & & & & & & & & & & & \\
\hline Residente & & & & & & & & & & & & \\
\hline Cirujano & & & & & & & & & & 册 & \# & \\
\hline
\end{tabular}

Consulta externa. Para el caso de la consulta externa se muestra que en todos los casos existe el cubrimiento y responsabilidad de un cirujano contratado por el hospital de lunes a viernes en el horario programado de 07:00 a 19:00 horas (Tablas 6 y 7 ).
Como se aprecia se mantiene una productividad de 3,04 pacientes/hora, por tanto no se puede afirmar que exista un aporte económico de las actividades de los internos y residentes, los estudiantes pueden incluso retrasar los procesos, sin embargo si hay una mejoría en la oportunidad y 
Tabla 5. Cuantificación económica del servicio de urgencias en el Hospital Universitario Hernando Moncaleano Perdomo, entre mayo de 2008 y abril de 2009.

\begin{tabular}{ll}
\hline \multicolumn{1}{c}{ Descripción } & \multicolumn{1}{c}{ Resultado } \\
\hline 1.216 egresos/añox4,21 días estancia/ 365 días/año & 14,02 pacientes/día \\
14 pacientes/día / 3 pacientes/hora AM & 4,7 horas/día \\
+ 2 horas revista de internos y residentes PM & 2,0 horas \\
Subtotal horas de revista/día & 6,7 horas \\
Hospital Universitario de Neiva & 6,7 horas \\
Universidad Surcolombiana & 0,0 horas \\
HUN: $\$ 42.500 \times 24$ horas $\times 365$ días/año & $\$ 372.300 .000,00$ \\
\hline USCO $\$ 42.500 \times 00,0$ horas & $\$ 00,00$ \\
\hline
\end{tabular}

Tabla 6. Cuantificación horaria del servicio de consulta externa del Hospital Universitario Hernando Moncaleano Perdomo, entre mayo de 2008 y abril de 2009.

\begin{tabular}{|c|c|c|c|c|c|c|c|c|c|c|c|c|}
\hline C. E. Cirugía/hora & 07 & 08 & 09 & 10 & 11 & 12 & 13 & 14 & 15 & 16 & 17 & 18 \\
\hline \multicolumn{13}{|l|}{ Interno } \\
\hline \multicolumn{13}{|l|}{ Residente } \\
\hline Cirujano & & & & & & & & & & & 弗 & \\
\hline
\end{tabular}

Tabla 7. Cuantificación económica del servicio de consulta externa en el Hospital Universitario Hernando Moncaleano Perdomo por año, entre mayo de 2008 y abril de 2009.

\begin{tabular}{ll}
\hline \multicolumn{1}{c}{ Descripción } & \multicolumn{1}{c}{ Resultado } \\
\hline 6.809 consultas /año $\times 3,04$ pacientes/hora & 2.239 horas/año \\
$2.239 /$ horas/año / 250 días hábiles/año & 8,95 horas/día \\
Hospital Universitario de Neiva & 2.239 horas \\
Universidad Surcolombiana & 0,0 horas \\
HUN: $\$ 42.500,00 \times 2.239$ horas/año & $\$ 95.157 .500,00$ \\
\hline USCO $\$ 42.500 \times 0,0$ horas/año & $\$ 0,00$ \\
\hline
\end{tabular}

en el diligenciamiento de papelería y especialmente de las contra-referencias a las Entidades Prestadoras de Salud.

Cirugía gastro-oncológica. Se confrontaron las horas de revista/día contratadas por el hospital con las actividades de los internos y residentes (Tablas 8 y 9).

E1 aporte de la USCO se cuantifica en \$ $62.050 .000,00$ al año que corresponde a las al menos 4 horas que representan las actividades 
Tabla 8. Cuantificación horaria del servicio de gastro-oncología en el Hospital Universitario Hernando Moncaleano Perdomo, entre mayo de 2008 y abril de 2009.

\begin{tabular}{|c|c|c|c|c|c|c|c|c|c|c|c|c|c|}
\hline Gastro/hora & 06 & 07 & 08 & 09 & 10 & 11 & 12 & 13 & 14 & 15 & 16 & 17 & 18 \\
\hline \multicolumn{14}{|l|}{ Interno } \\
\hline \multicolumn{14}{|l|}{ Residente } \\
\hline Cirujano & & & & & & & & & & & & & \\
\hline
\end{tabular}

Tabla 9. Cuantificación económica del servicio de gastro-oncología en el Hospital Universitario Hernando Moncaleano Perdomo, entre mayo de 2008 y abril de 2009.

\begin{tabular}{|c|c|}
\hline Descripción & Resultado \\
\hline HUN: 2 horas/ día x 365 días/año & 730 horas \\
\hline USCO 4horas/día x 365 días/año & 1.460 horas \\
\hline HUN: $\$ 42.500,00 \times 730$ horas/año & $\$ 31.025 .000,00$ \\
\hline USCO $\$ 42.500 \times 1.460$ horas/año & $\$ 62.050 .000,00$ \\
\hline
\end{tabular}

post-revista del cirujano de contrato y la revista de la tarde.

Cirugía de tórax. El cirujano de tórax tiene asignadas y contratadas 2 /horas de revista, el apoyo de internos y residentes (Tablas 10 y 11).
Como sucede con gastro-oncología, el aporte en la preparación de la revista, diligenciamiento de papelería y en las actividades post-revista de la mañana y la tarde se reflejan en al menos 4 horas/día que corresponden a \$62.050.000,00 al año.

Tabla 10. Cuantificación horaria del servicio de Cirugía de Tórax en el Hospital Universitario Hernando Moncaleano Perdomo, entre mayo de 2008 y abril de 2009.

\begin{tabular}{|c|c|c|c|c|c|c|c|c|c|c|c|c|c|}
\hline Tórax/hora & 06 & 07 & 08 & 09 & 10 & 11 & 12 & 13 & 14 & 15 & 16 & 17 & 18 \\
\hline Interno & & & & & & & & & & & & & \\
\hline Residente & & & & & & & & & & & & & \\
\hline Cirujano & & & & & & & & & & & & & \\
\hline
\end{tabular}

Tabla 11. Cuantificación económica del servicio de Cirugía de Tórax en el Hospital Universitario Hernando Moncaleano Perdomo, entre mayo de 2008 y abril de 2009.

\begin{tabular}{lc}
\hline Descripción & Resultado \\
\hline HUN: 2 horas/día x 365 días/año & 730 horas \\
USCO 4 horas/día x 365 días/año & 1.460 horas \\
HUN: $\$ 42.500,00 \times 730$ horas/año & $\$ 31.025 .000,00$ \\
\hline USCO $\$ \mathbf{4 2 . 5 0 0}$ x $\mathbf{1 . 4 6 0}$ horas/año & $\mathbf{\$ 6 2 . 0 5 0 . 0 0 0 , 0 0}$ \\
\hline
\end{tabular}


Grupo de Soporte Metabólico y Nutricional (GSMN). Adscrito al servicio de cirugía con la participación de nutricionista clínica y enfermera; responde a las interconsultas y manejo de todo el HUHMP, tanto de pisos como de UCI-A, incluye el aporte de internos y residentes (Tablas 12 y 13).
El HUN tiene contratado 4 horas/día, responde un promedio de 25 pacientes/día, los internos contribuyen al rendimiento horario preparando la revista, respuesta a interconsultas, diligenciamiento de formularios de medicamentos e insumos no POS, consideramos que un mínimo de 6 horas adicionales se dedican a este servicio, lo que representa \$ 93.075.000,00 al año (Tabla 14 y15).

Tabla 12. Cuantificación horaria del Grupo de Soporte Metabólico y Nutricional en el Hospital Universitario Hernando Moncaleano Perdomo, entre mayo de 2008 y abril de 2009.

\begin{tabular}{|c|c|c|c|c|c|c|c|c|c|c|c|c|c|}
\hline GSMN/hora & 06 & 07 & 08 & 09 & 10 & 11 & 12 & 13 & 14 & 15 & 16 & 17 & 18 \\
\hline Interno & & & & & & & & 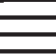 & & & & & \\
\hline Residente & & & & & & & & & & & & & \\
\hline Cirujano & & & & & & & & & & & & & \\
\hline
\end{tabular}

Tabla 13. Cuantificación económica del Grupo de Soporte Metabólico y Nutricional en el Hospital Universitario Hernando Moncaleano Perdomo, entre mayo de 2008 y abril de 2009.

\begin{tabular}{lc}
\hline \multicolumn{1}{c}{ Descripción } & Resultado \\
\hline HUN: 4 horas/día $\times 365$ días/año & 1.460 horas \\
USCO 6 horas/ día $\times 365$ días/año & 2.190 horas \\
HUN: $\$ 42.500,00 \times 1.460$ horas/año & $\$ 62.050 .000,00$ \\
\hline USCO $\$ 42.500 \times 2.190$ horas/año & $\$ 93.075 .000,00$ \\
\hline
\end{tabular}

Tabla 14. Cuantificación horaria de Clínica de heridas del Hospital Universitario Hernando Moncaleano Perdomo, entre mayo de 2008 y abril de 2009.

\begin{tabular}{|c|c|c|c|c|c|c|c|c|c|c|c|c|c|}
\hline Heridas / hora & 06 & 07 & 08 & 09 & 10 & 11 & 12 & 13 & 14 & 15 & 16 & 17 & 18 \\
\hline \multicolumn{14}{|l|}{ Interno } \\
\hline \multicolumn{14}{|l|}{ Residente } \\
\hline Cirujano & & & & & & & & & & & & & \\
\hline
\end{tabular}

Tabla 15. Cuantificación económica de Clínica de heridas del Hospital Universitario Hernando Moncaleano Perdomo, entre mayo de 2008 y abril de 2009.

\begin{tabular}{|c|c|}
\hline Descripción & Resultado \\
\hline HUN: 6 horas/día x 250 días / año & 1.500 horas \\
\hline USCO 1 horas/día x 250 días/año & 250 horas \\
\hline HUN: $\$ 42.500,00 \times 1.500$ horas/año & $\$ 63.750 .000,00$ \\
\hline USCO $\$ 42.500 \times 250$ horas/año & $\$ 10.625 .000,00$ \\
\hline
\end{tabular}


De reciente creación, ha tenido un crecimiento vertiginoso, para la fecha del estudio, contaba con el aporte adicional de 1 hora a la contratación del HUN que se resume en \$ 10.625.000,00 al año.

Cirugía programada y de urgencias. Se dispone de ayudantía quirúrgica permanente las 24 horas del día, los 365 días del año (Tabla 16 y 18).

Para cuantificar el aporte de los internos y residentes como ayudantes quirúrgicos fue necesario revisar la contratación y cuantificarlas a las tarifas contratadas para la época, ISS 2001 y SOAT 2009, se presenta como ejemplo las apendicetomías como primera cirugía (CX1) y el cuadro resumen total (Tabla 19).
Cirugía de cabeza y cuello. No se incluye dado el volumen de pacientes, a la irregularidad en la cantidad de pacientes y a que en general se suple con las actividades de los internos y residentes asignados a los otros servicios.

Cirugía de seno. Dado el volumen de pacientes y el manejo ambulatorio de los pacientes, son manejados directamente por el especialista en seno, con el aporte de los internos y residentes de los otros servicios, no se consideró aporte económico (Tabla 20).

El mínimo aporte de los internos y residentes de Cirugía General con las ayudantías quirúrgicas a tarifa ISS 2001 fue de \$ 601.734.830,00/año o a tarifa SOAT $2009 \$ 644.566 .046,00 /$ año.

Tabla 16. Cuantificación horaria Cirugía programada del Hospital Universitario Hernando Moncaleano Perdomo, entre mayo de 2008 y abril de 2009.

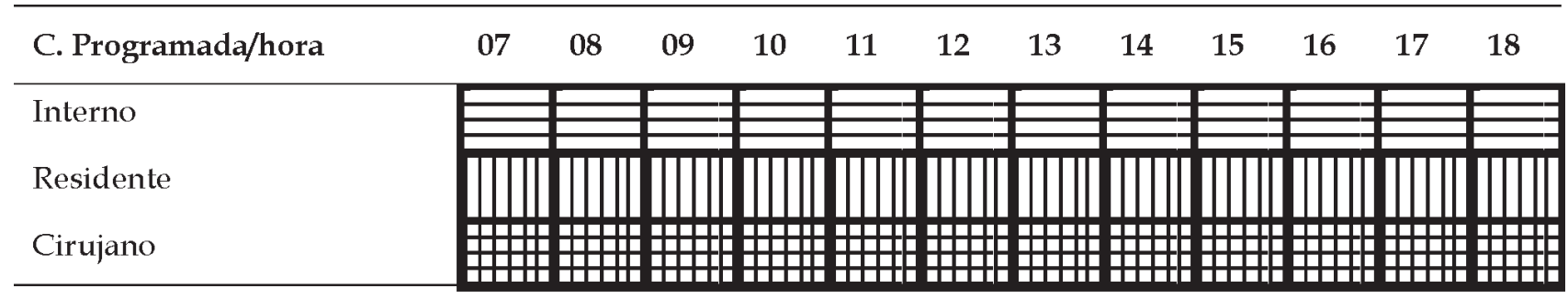

Tabla 17. Cuantificación horaria de Cirugía de urgencias del Hospital Universitario Hernando Moncaleano Perdomo, entre mayo de 2008 y abril de 2009.

\begin{tabular}{|c|c|c|c|c|c|c|c|c|c|c|c|c|}
\hline C. Urgencias / hora & 07 & 08 & 09 & 10 & 11 & 12 & 13 & 14 & 15 & 16 & 17 & 18 \\
\hline Interno & & & & & & & & & & & & \\
\hline Residente & & & & & & & & & & & & \\
\hline Cirujano & & & & & & & & & & & & \\
\hline
\end{tabular}

Tabla 18. Cuantificación económica de Cirugía de urgencias del Hospital Universitario Hernando Moncaleano Perdomo, entre mayo de 2008 y abril de 2009.

\begin{tabular}{|c|c|c|c|c|c|c|c|c|c|c|c|c|}
\hline C. Urgencias / hora & 19 & 20 & 21 & 22 & 23 & 24 & 01 & 02 & 03 & 04 & 05 & 06 \\
\hline \multicolumn{13}{|l|}{ Interno } \\
\hline \multicolumn{13}{|l|}{ Residente } \\
\hline Cirujano & & & & & & & & & & & & \\
\hline
\end{tabular}


Tabla 19. Cuantificación económica de ayudantías del Hospital Universitario Hernando Moncaleano Perdomo, entre mayo de 2008 y abril de 2009.

\begin{tabular}{lccccc}
\hline \multicolumn{5}{c}{ Ayudantías quirúrgicas Cirugía1 (Cx1) / Cirugía2 (Cx2) } \\
\hline \multicolumn{1}{c}{ Procedimiento } & UVR & V\$ & \$ ISS & \$ SOAT 2009 & Total \\
Apendicetomía & CX 1 & 2001 & SO & 34.560 & 498 \\
Apendicetomía+colocación dren & 80 & 360 & 28.800 & 34.560 & 3 \\
Apendicetomía+drenaje absceso & 80 & 360 & 28.800 & 34.560 & 3 \\
Apendicetomía+ peritonitis & 80 & 360 & 28.800 & 34.560 & 22 \\
Apendicetomía+laparotomía & 80 & 360 & 28.800 & 34.560 & 23 \\
Apendicetomía+resección ciego & 80 & 360 & 28.800 & 34.560 & 5 \\
Apendicetomía+hemicolectomía & 80 & 360 & 28.800 & 34.560 & 3 \\
\hline \multicolumn{1}{c}{ Apendicetomías } & & & 16.041 .600 & 19.249 .920 & 557 \\
\hline
\end{tabular}

Tabla 20. Resumen cuantificación económica del Departamento Cirugía del Hospital Universitario Hernando Moncaleano Perdomo, entre mayo de 2008 y abril de 2009.

\begin{tabular}{lcc}
\hline & Descripción & Resultado valor \$ \\
\hline USCO Revista de piso & $\$ 159.778 .750,00$ \\
USCO Consulta Externa & $\$$ & 00,00 \\
USCO Urgencias & $\$$ & 00,00 \\
USCO Cirugía de tórax & $\$ 62.050 .000,00$ \\
USCO Gastro-oncología & $\$ 62.050 .000,00$ \\
USCO GSMN & $\$ 93.075 .000,00$ \\
USCO Heridas & $\$ 10.625 .000,00$ \\
USCO Ayudantías quirúrgicas ISS 2001 & $\$ 214.156 .080,00$ \\
USCO Ayudantías quirúrgicas SOAT 2009 & $\$ 256.987 .296,00$ \\
\hline USCO Total ISS 2001 & $\$ \mathbf{6 0 1 . 7 3 4 . 8 3 0 , 0 0}$ \\
\hline USCO Total SOAT 2009 & $\$ \mathbf{6 4 4 . 5 6 6 . 0 4 6 , 0 0}$ \\
\hline
\end{tabular}

\section{DISCUSIÓN}

En estudios previos se ha demostrado que las prácticas Docentes-Asistenciales permiten de for- ma general a los centros asistenciales, disminuir el tiempo promedio de espera de los usuarios para recibir la atención asistencial lo que a su vez produce un aumento en la participación en el mercado, así como el fortalecimiento de la cultura de 
la calidad, derivado del conocimiento científico que se produce en las actividades diarias ${ }^{(10)}$.

Como se dijo, en el año 1998, en un trabajo del post-grado en Gerencia en Servicios de Salud y Seguridad Social, se buscaba valorar los "Aportes realizados por la USCO a través de las actividades docente asistenciales del programa de medicina, en la E.S.E. Hospital Departamental Hernando Moncaleano Perdomo Departamento de Clínicas. De octubre de 1997 a marzo de 1998, se revisaron las agendas del personal docente por los diferentes servicios, específicamente para Cirugía General se resume en \$ $31.857 .400,00$ en aporte del profesorado y en $\$$ 7.705.200,00 en ayudantías quirúrgicas realizadas por los residentes e internos por el semestre. Se aclara que para la fecha se contaba con 4 residentes pues apenas existían de I y II año ${ }^{(9)}$.

En estudios más recientes, se resalta que en el 2005 la Facultad de Medicina de la Universidad Surcolombiana contaba con 76 docentes (con especialidades clínicas), 57 internos y 27 residentes; El hospital con 89 médicos, de los cuales 34 tenían contrato laboral con ambas instituciones. Lo que cabe destacar es el hecho de que son los miembros de la USCO, según el estudio, quienes realizan el $83,13 \%$ de los procedimientos de las 20 especialidades médicas ofrecidas ${ }^{(8)}$.

Sin embargo el trabajo presenta objeciones metodológicas y además es sujeto de sesgos derivados de la cuantificación de las actividades de los docentes sin tener en cuenta los cruces o concurrencia de horarios.

El presente trabajo por tanto, específica el aporte de internos y residentes exclusivamente en el aspecto económico, no fue considerado como objetivo determinarlo en la calidad de atención de los pacientes ni el aspecto científico, investigativo, de producción intelectual y de prestigio institucional que de ello se deriva.

Se trato de una manera racional de describir y cuantificar las actividades de los diferentes actores, sin exagerar sus aportes o sobredimensionar sus valores.

Se define que el aporte de los internos y residentes de Cirugía General cuando se cuantifican las ayudantías quirúrgica a tarifa ISS 2001 corresponde a un total de $\$ 601.734 .830,00$ y a tarifa SOAT 2009 a \$ 644.566.046,00 que representa un aporte mensual promedio \$50.144.569,00 y \$53.713.837,00 respectivamente.
Se podría de toda manera objetar que no se tuvieron en cuenta los costos de la permanencia y sostenimiento de los internos y residentes como alimentación, lavandería, hotelería entre otros, sin embargo no fue el objetivo del trabajo y podría ser motivo de otra investigación.

\section{CONCLUSIONES}

No se discute, no fue la intensión ni el objetivo del presente estudio, el aporte en calidad, oportunidad, rendimiento, eficiencia y eficacia del aporte de los internos y residentes de Cirugía General de la USCO al HUN.

El aporte en desarrollo investigativo y tecnológi$\mathrm{co}$, el reconocimiento institucional derivado de trabajos y publicaciones, la representación en congresos nacionales e inter nacionales y el posicionamiento como entidad de alta complejidad, tampoco se discuten.

El mayor aporte económico se deriva de las ayudantías quirúrgica que representan un mínimo del 35,6\% a tarifa ISS 2001 y $39,9 \%$ a tarifa SOAT 2009.

En segundo lugar, se encuentra la revista de piso con un $26,6 \%$ y $24,8 \%$ cuando se cuantifica el total a tarifas ISS 2001 y SOAT 2009 respectivamente.

El tercer lugar lo ocupa el aporte al Grupo de Soporte Metabólico y Nutricional con el 10,3\% y 9,6\% a tarifas ISS 2001 y SOAT 2009.

En cuarto y quinto lugar están las subespecialidades de cirugía gastro-oncológica y cirugía de tórax 10,3\% y 9,6\% y en sexto lugar la Clínica de heridas con el 1,8\% y $1,6 \%$.

Las ayudantías quirúrgicas y la revista de piso suman más del 50\% del total del aporte total del aporte con el $66,5 \%$ y $64,7 \%$ respectivamente.

Para futuras comparaciones, teniendo en cuenta que para el año 2009 el salario mínimo legal mensual vigente (SMLV) era de \$ 496.900,00 el aporte corresponde a 1.210 SMLV a tarifas ISS 2001 y a 1.297 SMLV a tarifa SOAT 2009.

De no contarse con este recurso, el HUN se vería obligado a contratar al personal de especialistas y médicos generales que suplan estas necesidades y garanticen el cumplimiento de los estándares de calidad. 
Los internos no reciben, por su trabajo, ninguna contraprestación económica del Estado, los residentes reciben 3 salarios mínimos legales mensuales vigentes (SMLV) de forma trimestral, por cada año de residencia que corresponde a $\$ 1.490 .700,00$ mensuales condonables al graduarse con una nota promedio no inferior a 3,5, mediante el crédito beca ICETEX-Ministerio de la Protección Social.

Nuevos estudios en este y otros sentidos podrán en el futuro ser desarrollados para enriquecer la discusión y controvertir muchos aspectos a la luz de las realidades individuales, locales, regionales, nacionales y mundiales.

Consideramos que el Hospital Universitario de Neiva debe ver en la relación Docencia-Servicio con la Universidad Surcolombiana una fortaleza y una oportunidad y no una amenaza o una debilidad.

\section{REFERENCIAS}

1. Custer, W.S., Willke, R.J. 1991. Teaching hospital costs: The effects of medical staff characteristics. Health Services Research 25(6):831-857.

2. Smet, M. 2002. Cost characteristics of hospitals. Social Science and Medicine 55(6):895-906.

3. Cleverley, WO. 2002. The hospital cost index: a new way to assess relative cost-efficiency. Healthcare Financial Management 56(7):36-42.

4. Centro de Investigaciones para el Desarro1lo-CID. COMPONENTE ECONÓMICO FINANCIERO. Facultad de Ciencias Econó- micas. Universidad Nacional de Colombia. En: www.cid.unal.edu.co. 2006. Consulta: noviembre de 2010.

5. Agudelo, C.A., Sánchez C., Robledo R., et al. Modelo académico de Hospital Universitario En: Biblioteca Digital Universidad Nacional, http:/ / www.bdigital.unal.edu.co/ 643/1/01_Modelo_Academico_HU.pdf. 46p.; consulta: noviembre de 2010.

6. Decreto 1011 de 2006 SOGC. Ministerio de la Protección Social. Por el cual se establece el Sistema Obligatorio de Garantía de Calidad de la Atención de Salud del Sistema General de Seguridad Social en Salud. En: www. minproteccionsocial.gov.co 18p.; consulta: noviembre de 2010.

7. Decreto 190 de 1996. Por el cual se dictan normas que reglamentan la relación Docente-Asistencial en el sistema general de seguridad social en salud. En: www. minproteccionsocial. gov.co 18p.; consulta: noviembre de 2010.

8. Acevedo, J.J., Corredor, G.I., Rivera, L. et al. Cuantificación de la productividad de las actividades realizadas por docentes, residentes e internos de Medicina de la Universidad Surcolombiana en el Hospital Universitario de Hernando Moncaleano Perdomo de Neiva-Huila durante el mes de febrero de 2004. Tesis 2004.

9. Convenio Docencia-Asistencia, Hospital Universitario de Neiva- Universidad Surcolombiana, 2 de agosto de 1999.

10. Cezar, A.M. 2001. Los hospitales universitarios: pasado, presente y futuro. Cuadernos Médico Sociales 79:119-128. 


\title{
POSGRADOS CLÍNICOS
}

Universidad

Surcolombiana

\section{ESPECIALIZACIÓN EN PEDIATRÍA}

Registro ICFES No. 111456180924100111400

Creada mediante acuerdo Consejo Superior Universitario No. 034 del 29-05-1996

\author{
Denominación Académica: Programa de Especialización en Pediatría \\ Modalidad: \\ Presencial \\ Duración: \\ 3 años (6 semestres) \\ Cupos: \\ 3 Anual \\ Título: \\ Especialista en Pediatría
}

\section{ESPECIALIZACIÓN EN CIRUGÍA GENERAL}

Registro ICFES No. 111456170004100111400

Creada mediante acuerdo Consejo Superior Universitario No. 035 del 29-05-1996

$\begin{array}{ll}\text { Denominación Académica: } & \text { Programa de Especialización en Cirugía General } \\ \text { Modalidad: } & \text { Teórico - Presencial } \\ \text { Duración: } & 4 \text { años (8 semestres) } \\ \text { Cupos: } & 2 \text { Anuales } \\ \text { Título: } & \text { Especialista en Cirugía General }\end{array}$

\section{ESPECIALIZACIÓN EN GERENCIA DE SERVICIOS DE SALUD Y SEGURIDAD SOCIAL}

Creada mediante acuerdo Consejo Superior Universitario No. 021 del 10-07-1995 SNIES No. 3503, Registro calificado de calidad Res. No 450 de febrero 5 de 2008 Ministerio de Educación Nacional.

Denominación Académica: Programa de Especialización en Gerencia de Servicios de salud y Seguridad Social

Modalidad: Semipresencial

Duración: 3 semestres

Cupos: 25 Anuales

Título:

Especialista en Gerencia de Servicios de salud y Seguridad Social 